J. Lake Sci. (湖泊科学) , 2017, 29(6): 1485-1490

DOI 10. 18307/2017. 0620

(c) 2017 by Journal of Lake Sciences

\title{
基于实测高光谱数据的鄱阳湖湿地植被光谱差异波段提取”
}

\author{
况润元, 曾 帅,赵哲, 肖阳 \\ (江西理工大学建筑与测绘工程学院, 赣州 341000)
}

\begin{abstract}
摘 要: 高光谱遥感技术的出现为有效解决湿地植被种类的精细识别和分类提供了可能. 通过实地测取鄱阳湖湿地 5 种 植被的高光谱数据, 在对数据预处理的基础上, 提出一种基于数据误差范围和植被光谱均值差的植被光谱差异波段提取 方法. 将该方法应用于包络线变换前后的光谱曲线提取植被的光谱差异波段,最后利用马氏距离法检验植被识别效果. 结果表明: 本文中的方法有效提取了植被光谱差异波段, 其中变换前光谱差异波段分别为 $663 \sim 688 \mathrm{~nm}$, 变换后为 $581 \sim$ 636、660 695 和 1225 1236 nm. 在光谱差异波段范围内, 同种植被的马氏距离值小于异种植被的马氏距离值, 可有效对 植被进行识别. 研究结果为湿地植被分类识别奠定了理论基础, 同时为湖泊湿地植被以及湖泊生态环境的保护决策提供 科学依据.
\end{abstract}

关键词: 鄱阳湖湿地;植被光谱;光谱差异波段

\section{Extraction of the discriminative bands of Lake Poyang wetland vegetation based on the measured hyperspectral data}

\section{KUANG Runyuan, ZENG Shuai, ZHAO Zhe \& XIAO Yang}

(School of Architectural and Surveying \& Mapping Engineering, Jiangxi University of Science and Technology, Ganzhou 341000, P.R.China)

Abstract: Identification and classification of wetland vegetation by spectral data is often one of the key points and difficulties in vegetation remote sensing. Compared with multispectral data, hyperspectral ones have the characteristics of more bands, larger amount of data and higher redundancy. The emergence of hyperspectral remote sensing technology provides chance for solving problems of identifying and classifying wetland vegetation species precisely. For the discrimination of wetland vegetation species with hyperspectral remote sensing technology, the extraction of the discriminative bands is very important. The hyperspectral data on Acorus calamus, Cynodon dactylon, Carex cinerascens, Persicaria hydropiper, Phalaris arundinacea in Lake Poyang wetland are measured by using ASD FieldSpec 4 spectrometers. A new method is proposed to extract the discriminative bands between vegetation species of original spectra and envelope spectra. Finally, we use the method of Mahalanobis distance to examine the vegetation identification effects. The research results show that: The new method can extract the discriminative bands between vegetation species effectively. The discriminative bands of original spectra is $663-688 \mathrm{~nm}$, and the discriminative bands of envelope spectra are : 418-451 $\mathrm{nm}, 487-507 \mathrm{~nm}, 621-637 \mathrm{~nm}, 658-85 \mathrm{~nm}, 1222-1245 \mathrm{~nm}$. In those discriminative bands, the Mahalanobis distance values between the same species is less than those among different species, so it could help us to identify different types of vegetation species effectively. The research results lay a foundation for the wetland vegetation classification, and provide a scientific basis for the protection of the lake wetland vegetation and the ecological environment of lakes.

Keywords: Lake Poyang wetland; hyperspectral data; discriminative bands

高光谱数据容易获取地物的局部精细信息,对光谱细节特征具有良好的表现能力,在分析植被的反射 光谱差异性有较大的潜力 ${ }^{[1]}$. 运用高光谱技术对湿地植被光谱进行识别分析是近些年来研究的热点和重点

* 国家自然科学基金项目(41101322)、江西省自然科学基金项目(20114BAB213022) 和江西省教育厅科学技术研究 项目 (GJJ160617) 联合资助. 2016-07 - 16 收稿; 2017-03-09 收修改稿. 况润元 (1976 ), 男,博士, 副教授; E-mail: rykuang@163.com. 
之一. 在光谱识别研究中, 不同类型地物可根据地物特有光谱特征进行有效区分, 这些光谱特征包括吸收位 置、光谱值、反射曲线深度、宽度与强度、峰谷位置以及斜率等 ${ }^{[2]}$. 相关专家和学者在植被光谱分析识别方面 的研究成果如下: 刘波等 ${ }^{[3]}$ 依据雅江源区地面实测高光谱数据, 分析高寒区草地光谱特征和退化过程中的 光谱响应变化; 任广波等 ${ }^{[4]}$ 基于黄河三角洲 6 种典型植被的实测光谱数据, 分析反射光谱特征并篮选出了 植被光谱差异波段; 安如等 ${ }^{[5]}$ 分析了三江源中东部草原区常见草种的反射光谱特征, 提取了典型草地植被 的光谱特征; 柴颖等 ${ }^{[6]}$ 利用美国 Sacramento-San Joaquin 三角洲植被的实测高光谱数据, 在对光谱特征分析 的基础上对湿地植被进行了分类; 刘克等 ${ }^{[7]}$ 测定野鸭湖湿地典型植被群落的高光谱数据, 分析提取了可用 于植被分类的光谱识别波段.

纵观国内外研究现状, 涉及到旱地、盐沼植被高光谱方面的研究较多, 在内湖淡水湿地植被光谱分类方 面的成果相对较少, 已有的关于植被高光谱反射特征知识有待进一步研究; 相对于旱地植被和盐沼植被, 湖 泊湿地植被受湖区水位影响较大, 光谱不仅具有高度相似性和空间变异性, 且具有较强的时间动态性; 针对 淡水湖泊湿地植被光谱分类识别的相关研究还不多见, 在植物种类精细分类识别方法上还有进一步研究空 间. 本文以鄱阳湖湿地 5 种优势种植被为研究对象, 通过分析湿地植物光谱特征, 提出一种新的植物光谱差 异波段提取方法,对湖泊湿地植被的种类识别方法方面进行探讨.

\section{1 研究区域与数据方法}

\section{1 研究区概况}

鄱阳湖湿地 $\left(28^{\circ} 22^{\prime} \sim 29^{\circ} 45^{\prime} \mathrm{N}, 115^{\circ} 47^{\prime} \sim 116^{\circ} 45^{\prime} \mathrm{E}\right)$ 位于中国江西省北部、长江中下游南岸, 是我国第一 大淡水湖泊生态湿地. 鄱阳湖承接赣江、饶河、信江、修河、抗河五河之水, 与长江相通, 流域内草洲、泛滥地、 河滩地大量分布且面积广阔. 整个流域内孕育着大量宝贵的物种资源, 其中的水生植被起着至关重要的作 用, 具有固碳、降解有毒物质、改善环境等功能. 作为初级生产者, 湿地植被是食草性鱼类主要的食物来源, 同时也是各种水生动物和越冬的候鸟活动栖息之地, 湿地内多种植被更是人类进行经济活动重要的生产资 料. 随着近几年气候变化和各种因素的影响, 鄱阳湖和其他内陆湖泊一样面临着水量调蓄能力下降、湖泊环 境污染加重、生物资源退化等问题 ${ }^{[8]}$. 鄱阳湖生态功能对整个长江中下游地区经济发展、自然资源的保护和 利用的重要意义不言而喻. 如何对鄱阳湖湿地中物种资源, 特别是植被资源进行科学保护已然成为一项迫 不及待的任务之一.

\section{2 数据采集及预处理}

采集地点为鄱阳湖南矶乡自然保护区, 采集时间为 2016 年 4 月 $18 、 19$ 日和 11 月 3 日, 天空晴朗无云或 局部少云, 此时湖区水位较低, 植物长势良好, 与其他季节相比, 植被光谱在此时相似度较高、分类难度较大. 利用美国 ASD 公司生产的 FieldSpec 4 便携式地物光谱仪采集植被高光谱数据. 光谱采样的波长范围为 $350 \sim 2500 \mathrm{~nm}$, 其中 $350 \sim 1000 \mathrm{~nm}$ 光谱分辨率为 $3 \mathrm{~nm} 、 1000 \sim 2500 \mathrm{~nm}$ 为 $10 \mathrm{~nm}$, 光谱采样间隔 $350 \sim 1000 \mathrm{~nm}$ 为 $1.4 \mathrm{~nm} 、 1000 \sim 2500 \mathrm{~nm}$ 为 $2 \mathrm{~nm}$, 数据采样间隔为 $1 \mathrm{~nm}$, 光纤前视场角为 $25^{\circ}$. 测量的植被有菖蒲 (Acorus calamus)、狗牙根 (Cynodon dactylon)、苔草 (Carex cinerascens)、水蓼 (Persicaria hydropiper)、曧草 (Phalaris arundinacea $) 5$ 种植被. 在植被光谱测量过程中, 选取植被单一、长势良好、植被盖度较高的植丛布置样方, 样 方大小设置为 $1 \mathrm{~m} \times 1 \mathrm{~m}$, 样方之间的距离大于 $3 \mathrm{~m}$, 为使数据具有代表性, 采样点均匀分布于研究区. 为保证 良好的光照条件, 测量时间选定在 10:00-14:30, 测量过程中每隔最多 $20 \mathrm{~min}$ 需进行一次白板优化, 测量前 需提前开机预热 $30 \mathrm{~min}$. 所有的测量数据均采用垂直观测法, 距离植被冠层上方 $1 \mathrm{~m}$ 左右测取数据, 每个采 样点测取 10 条光谱曲线, 每种植物测量 30 个样点, 随机抽取 10 个样点数据用以实验分析, 剩下的 20 个样 点数据对本文中提出的方法进行识别效果检验. 本文使用协方差来衡量每种植被样点的离散程度, 5 种植被 30 个样点之间的协方差平均值分别为 0.0208 (菖蒲)、 0.0219 (狗牙根)、 0.0321 (水蓼) 、 0.0344 (苔草) 和 0.2941 (鶧草).

植被反射光谱曲线采集后, 室内利用仪器自带的 ViewSpecPro 软件对植被光谱曲线进行以下预处理: (1)剔除异常光谱数据. 受光照及野外环境因素影响, 波谱曲线在测量过程中难免存在有偏差程度较大的异 常数据, 本文参照王锦地等 ${ }^{[9]}$ 所提到的强度大小检验法对数值偏差较大的数据进行剔除. (2)去除噪声波段. 
去除水汽噪声波段 $1350 \mathrm{~nm}+$ 及边缘效应噪声波段 350 400 nm, 所以本次实验截取的波段范围为 $400 \sim 1350$ $\mathrm{nm}$. (3)数据平滑去噪处理. 本文采用移动平均法对所测植被光谱曲线进行平滑去噪处理 ${ }^{[10]}$. (4) 对数据求取 均值光谱曲线并导出到 Execl 中求取数据的误差范围, 以便后续的植被光谱特征分析和提取植被光谱差异 波段.

\section{3 光谱变换方法}

已有研究表明:利用包络线法 (Continuum Removal, $C R$ ) 在一定程度上可以放大植被光谱间的差异性, 有效消除由噪声引起的误差, 有效突出光谱曲线的吸收和反射特征, 有利于植被光谱分类识别研究 ${ }^{[11]}$. 其 中包络线变换公式如 (1) (3) 表示.

$$
\begin{gathered}
C R_{i}=R_{i} / R_{H i} \\
R_{H i}=R_{\text {start }}+k\left(\lambda_{i}-\lambda_{\text {start }}\right) \\
k=\left(R_{\text {end }}-R_{\text {start }}\right) /\left(\lambda_{\text {end }}-\lambda_{\text {start }}\right)
\end{gathered}
$$

式中, $C R_{i}$ 为波段 $i$ 处的包络线值; $R_{i}$ 为波段 $i$ 处的光谱反射率; $R_{H i}$ 为波段 $i$ 处的 “外壳” 值; $R_{\text {star }}$ ～$R_{\text {end }}$ 分别为吸 收起点和终点处所对应的反射率; $\lambda_{\text {star }}$ 、 $\lambda_{\text {end }}$, $\lambda_{i}$ 分别为吸收起点、终点和波长 $i$ 处对应的波长值; $k$ 为吸收起、 终点之间的“外壳”斜率.

\section{4 光谱差异波段选择}

基于植被光谱特征分析，选择植被光谱差异波段是对植被进行分类识别的方法之一,主要可归类为基 于光谱特征 ${ }^{[4,12]}$ 和基于统计分析方法 ${ }^{[2-3,13]}$ 分析. 由于湿地植被光谱具有较强的空间变异性和时间动态性, 前者对光谱差异性较小的波段容易错提、漏提,植被识别效果受区域因素制约较明显; 基于统计学分析法在 一定程度上可有效降低外界因素的影响, 拥有较好的识别效果, 其中应用较为广泛的有均值置信区间带 法 $^{[13]}$. 高光谱数据具有波段数目较多, 数据圥余程度大, 如何对数据进行降维处理和相关变换对于光谱差 异波段的选择尤为重要 ${ }^{[14]}$. 本文提出了一种基于数据误差范围和植被光谱均值差的植被光谱差异波段提 取方法, 与前人提出的方法不同的是, 本方法能捕捉到植被光谱间反射率值的微小差异, 在保留植被光谱物 理意义的同时提取出植被光谱差异波段,在方法操作上方便快捷.

在光谱测量过程中, 由于外界因素的影响造成光谱数值在一定范围内波动, 这个数值波动范围即误差 范围, 我们用统计学中的置信水平来衡量这个误差范围的可靠程度 ${ }^{[15]}$. 置信水平表示样本统计值的精确 度, 它是指样本统计值落在参数值某一区域内的概率. 本文方法的基本思路为: 对于两种植被 $a 、 b$, 设定在 光谱波段 $i$ 处, 若两种植被的光谱数据均值差之绝对值大于两种植被光谱数据在 0.95 置信水平下的误差范 围值之和,则便可认为在波段 $i$ 处两种植被存在明显的差异性,即波段 $i$ 为两者可相互区别的光谱差异波段. 最后,将所有植被两两间的光谱差异波段进行交集运算,即可得到同时有效区分 5 种植被光谱的光谱差异 波段. 此外, 为方便后期实测高光谱数据与高光谱遥感遥感影像结合, 规定所选出的光谱差异波段范围必须 大于高光谱遥感数据光谱分辨率 (10 $\mathrm{nm}$ 左右). 其公式分别为:

$$
\begin{gathered}
\left|\overline{X_{a(i)}}-\overline{X_{b(i)}}\right|>V_{a(i)}+V_{b(i)} \\
V_{a(i)}=T_{(1-\alpha, n-1)} \cdot \operatorname{Ste}_{a(i)} \cdot n^{1 / 2} \\
V_{b(i)}=T_{(1-\alpha, n-1)} \cdot S t e_{b(i)} \cdot n^{1 / 2}
\end{gathered}
$$

式中, $\overline{X_{a(i)}} 、 \overline{X_{b(i)}}$ 分别为植被 $a 、 b$ 在波段 $i$ 处的均值光谱; $V_{a(i)} 、 V_{b(i)}$ 分别为植被 $a 、 b$ 在波段 $i$ 处的误差范围; $n$ 为样本数; $T$ 为 $t$ 分布值, 与置信水平参数 $\alpha$ 和样本数 $n$ 有关, 在 Execl 中可用 Tinv 函数求取, 本实验中取 $\alpha$ 值为 $0.95 ; S t e_{a(i)} 、 S t e_{b(i)}$ 分别为植被 $a 、 b$ 在波段 $i$ 处的标准差.

\section{5 识别效果分析方法}

马氏距离由印度统计学家 P.C. Mahalanobis 提出, 用以表示数据的协方差距离, 是一种计算两个未知样 本集之间相似度的有效方法 ${ }^{[16]}$, 马氏距离具有既消除变量间的相关性干扰且不受量纲的影响等优点 ${ }^{[17]}$, 其 中马氏距离公式为:

$$
D_{k}=\left(X_{k}-\mu\right)^{T} \cdot S^{-1}\left(X_{k}-\mu\right)
$$

式中, $D_{k}$ 为不同植被光谱曲线在 $k$ 波段处的马氏距离; $X_{k}$ 为不同光谱曲线在 $k$ 波段处的差值矩阵; $\mu$ 为零点 向量矩阵; $T$ 为矩阵的转置; $S^{-1}$ 为协方差矩阵逆矩阵. 
运用马氏距离法评价本文提出的方法对 5 种植被的识别效果. 首先计算每种植被 20 个验证样点的光 谱平均值, 然后以任一种植被为比较植被, 分别计算每种植被每个验证样点与比较植被的平均值之间的马 氏距离, 然后计算这 20 个样点的马氏距离平均值, 最后对所有光谱差异波段内的马氏距离值求算术平均值 作为最终的马氏距离值. 如果不同植被间的马氏距离大于同种植被的马氏距离, 则说明所提取的光谱差异 波段能有效识别不同植被,进而说明本文提出的方法具有可行性.

\section{2 结果与讨论}

\section{1 光谱分析及差异波段选择}

图 1 为植被的原始反射光谱, 5 种植被曲线都表现出植被特有的曲线特征. 受植被本身生化组分和冠层 结构的影响, 植被光谱曲线表现出细微的差别, 通过对光谱分析识别不同植被间的细小差异, 实现对植被种 类的精细分类 ${ }^{[18]}$. 在可见光波段, 5 种植被的光谱曲线上都表现为低反射率, 其中在蓝光 $(400 \sim 530 \mathrm{~nm})$ 和 红光 (550 730 $\mathrm{nm}$ ) 波段吸收较为强烈, 形成蓝谷和红谷 2 个吸收谷, 介于两者间的绿光波段则形成 1 个凸 起的反射峰. 在波段 670 740 $\mathrm{nm}$ 的 “红边” 反射率迅速增高, 增长率大约为 40\% 50\%, 780 950 nm 范围内 继续保持高反射率值. 在近红外波段, 以 $960 、 1200 \mathrm{~nm}$ 为中心形成了水分的吸收带, 形成了吸收谷. 根据本 文提出的方法,提取出的光谱差异波段为 $663 \sim 688 \mathrm{~nm}$,此区间处于光谱曲线的红光波段.

通过对原始光谱的分析, 可以看出 5 种湿地植被的反射光谱曲线有 4 个较为明显的吸收谷, 分别为 $400 \sim 530 \mathrm{~nm}$ 的蓝光波段、550 730 nm 的红光波段、930 1000 nm 的水吸收波段以及 $1100 \sim 1250 \mathrm{~nm}$ 的水和 氧吸收波段, 对这 4 个吸收谷进行包络线光谱计算(图 2). 湿地植被在 4 个吸收波段具有不同的吸收强度, 第二波段吸收强度最大, 其次为第一波段, 在近红外的第三波段吸收强度最弱. 苔草和菖蒲在第一波段的吸 收强度较大, 而狗牙根、水蓼和曧草的相对较小. 5 种植被在第二波段强度都很大, 而在第三波段中吸收深度 都小于 0.1 ( 深度值 $=1-C R_{i}$ ), 第四波段中吸收强度也较小, 其中曧草的强度最大而狗牙根的最小. 根据本文 提出的方法, 提取出的光谱差异波段有 418 451、487 507、621 637、658 685 和 1222 1245 nm. 其中 418 451 和 487 507 nm 对应第一吸收谷, 621 637 和 658 685 nm 对应第二吸收谷, 1222 1245 nm 对应第四吸 收谷.

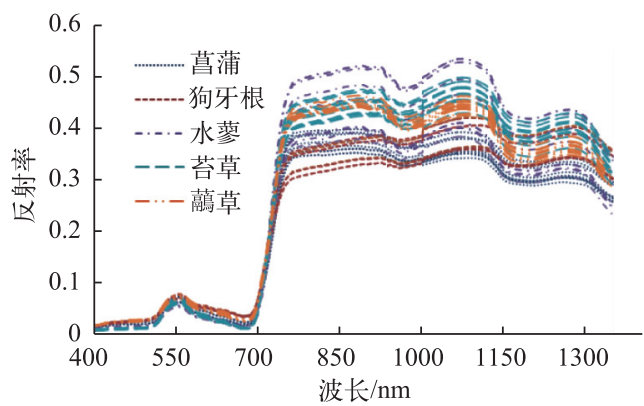

图 1 植被的反射光谱

Fig.1 The spectrum of vegetation species

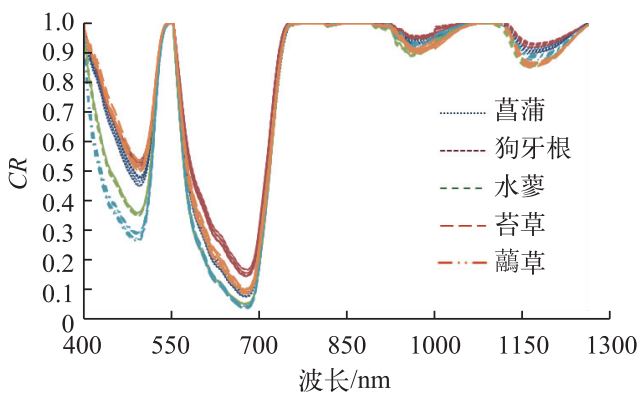

图 2 植被包络线光谱

Fig. 2 The spectrum of vegetation species after transforming

\section{2 识别结果分析}

利用马氏距离法检验特征波段对 5 种植被的识别效果, 分别计算 5 种植被的马氏距离值(表 1), 其中对 角线右上方为变换前植被在光谱差异波段范围内 $(663 \sim 688 \mathrm{~nm})$ 的马氏距离值, 左下方为包络线变换后的 马氏距离值, 对角线括号外是变换前同种植被的马氏距离值, 括号内是变换后同种的马氏距离值. 无论是变 换前还是变换后, 在对应的光谱差异波段范围内, 同种植被间的马氏距离值均小于异种植被间的马氏距离 值, 不同植被差异显著, 识别效果明显, 且变换后异种植被的马氏距离较变换前数值有所增大, 植被间的差 异更加明显. 以上分析表明, 本文中所提出的光谱差异波段提取方法可有效用于植被光谱识别; 通过包络线 变换后, 在光谱差异波段范围内不同植被光谱差异性更加明显, 识别效果更佳. 
表 15 种植被光谱差异波段马氏距离值

Tab.1 The Mahalanobis Distance of five vegetation species discriminative spectrum

\begin{tabular}{cccccc}
\hline \multirow{2}{*}{ 植被 } & \multicolumn{5}{c}{ 马氏距离值 } \\
\cline { 2 - 6 } & 菖蒲 & 狗牙根 & 水䓹 & 苔草 & 曧草 \\
\hline 菖蒲 & $2.208(2.567)$ & 2.217 & 2.527 & 2.237 & 2.461 \\
狗牙根 & 3.440 & $2.100(2.004)$ & 2.184 & 2.177 & 2.140 \\
水蓼 & 3.967 & 3.848 & $2.168(1.953)$ & 2.249 & 2.217 \\
苔草 & 3.934 & 3.908 & 3.475 & $2.108(2.381)$ & 2.182 \\
曧草 & 3.167 & 2.882 & 3.902 & 3.841 & $2.131(2.432)$ \\
\hline
\end{tabular}

\section{3 结论}

本文利用高光谱技术实地测取了鄱阳湖 5 种植被的高光谱数据, 针对湖泊湿地植被光谱相似度高、难 以分类识别等特点, 提出了一种基于数据误差范围和植被光谱均值差的植被光谱差异波段提取方法, 对植 被光谱包络线变换前后的曲线进行了分析并提取出了 5 种植被的光谱差异波段,最后运用马氏距离法检验 了该方法的识别效果. 得出以下结论:

马氏距离法较好地检验了本文所提出方法的有效性, 利用所提出的方法能有效提取出可以识别鄱阳湖 湿地 5 种典型植被的光谱差异波段, 同时达到了高光谱降维的目的,其中 5 种植被原始光谱曲线的差异波段 为 $663 \sim 688 \mathrm{~nm}$, 包络线光谱曲线的差异波段为 418 451、487 507、621 637、658 685 和 1222 1245 nm. 在 光谱差异波段范围内, 同种植被间的马氏距离值小于异种植被间的马氏距离值, 植被间的光谱差异显著. 与 原始光谱曲线相比,通过包络线变换后植被在光谱差异波段范围内的差异性更加明显.

地物光谱识别是高光谱遥感地物属性识别分类的基础, 不同类型地物可根据某些光谱差异显著的波段 进行有效识别, 如何对这些光谱差异波段范围进行界定和篮选则需要一定的方法, 本文提出的方法正可解 决了这一问题. 在通过对植被光谱差异波段进行选取, 有利于卫星传感器波段选择, 可以更好地应用于多光 谱或高光谱影像数据分类研究. 研究成果为湿地遥感影像解译、湿地植被制图奠定了理论基础, 同时在方法 上也为淡水湖泊湿地植被光谱识别分类提供借鉴意义. 本文重点在于植被光谱差异波段提取方法研究, 本 次实验测取了鄱阳湖丰水期前 5 种植被光谱数据并提取了光谱差异波段, 其他季节还会继续跟进深人研究. 此外, 对于如何利用地面实测光谱数据结合相应的遥感影像数据进行应用分析也是未来的研究重点.

\section{4 参考文献}

[ 1 ] Zomer RJ, Trabucco A, Ustin SL. Building spectral libraries for wetlands land cover classification and hyperspectral remote sensing. Journal of Environmental Management, 2009, 90(7) : 2170-2177.

[ 2 ] He Mingyi, Chang Wenjuan, Mei Shaohui. Advance in feature mining from hyperspectral remote sensing data. Spacecraft Recovery \& Remote Sensing, 2013,34(1): 1-12. [何明一, 畅文娟, 梅少辉. 高光谱遥感数据特征挖掘技术研究进展. 航天返回与遥感, 2013,34(1): 1-12.]

[ 3 ] Liu Bo, Shen Weishou, Li Ru et al. Spectral characteristics of alpiine grassland during degradation process in the source region of Yarlung Zangbo. Spectroscopy and Spectral Analysis, 2013, 33(6): 1598-1602. [刘波, 沈渭寿, 李儒等. 雅鲁 藏布江源区高寒草地退化光谱响应变化研究. 光谱学与光谱分析, 2013, 33(6): 1598-1602.]

[ 4 ] Ren Guangbo, Zhang Jie, Ma Yi. Spectral discrimination and separable feature lookup table of typical vegetation species in Yellow River Delta wetland. Marine Environmental Science, 2015, 34(3) : 420-426. [任广波, 张杰, 马毅. 黄河三角洲 典型植被地物光谱特征分析与可分性查找表. 海洋环境科学, 2015, 34(3): 420-426.]

[ 5 ] An Ru, Jiang Danping, Li Xiaoxue et al. Using hyperspectral data to determine spectral characteristics of grassland vegetation in central and eastern parts of Three-River source. Remote Sensing Technology and Application, 2014, 29(2) : 202211. [ 安如, 蒋丹萍, 李晓雪等. 基于地面实测高光谱数据的三江源中东部草地植被光谱特征研究. 遥感技术与 应用, 2014, 29(2) : 202-211.] 
[ 6 ] Chai Ying, Ruan Renzong, Fu Qiaoni. Extraction of wetland vegetation information using hyperspectral image data. Journal of Nanjing Forestry Univerity: Natural Sciences Edition, 2015, 39(1) : 181-184. [柴颖, 阮仁宗, 傅巧妮. 高光谱数据 湿地植被类型信息提取. 南京林业大学学报: 自然科学版, 2015, 39(1) : 181-184.]

[ 7 ] Liu Ke, Zhao Wenji, Guo Xiaoyu et al. Spectral bands of typical wetland vegetation in Wild Duck Lake. Acta Ecologica Sinica, 2010, 30(21): 5853-5861. [刘克, 赵文吉, 郭逍宇等. 野鸭湖典型湿地植物光谱特征. 生态学报, 2010, 30(21): 5853-5861.]

[ 8 ] Yang Guishan, Ma Ronghua, Zhang Lu et al. Lake status, major problems and protection strategy in China. J Lake Sci, 2010, 22(6) : 799-810. DOI: 10.18307/2010.0601. [杨桂山, 马荣华, 张路等. 中国湖泊现状及面临的重大问题与 保护策略. 湖泊科学, 2010, 22(6): 799-810.]

[ 9 ] Wang Jindi, Zhang Lixin, Liu Qinhuo et al eds. Chinese typical feature spectrum knowledge base. Beijing: Science Press, 2008: 71-73. [王锦地, 张立新, 柳钦火等. 中国典型地物波谱知识库. 北京: 科学出版社, 2008: 71-73.]

[10] Zhao Zhao. Analysis of spectral features based on water content of Xinjiang Desert Plants[Dissertation]. Urumqi: Xinjiang Agricultural University, 2011. [ 赵钊. 新疆荒漠植物含水率高光谱特征分析 [ 学位论文]. 乌鲁木齐: 新疆农业大 学, 2011.]

[11] Schmidt KS, Skidmore AK. Spectral discrimination of vegetation types in coastal wet land. Remote Sensing of Environment, $2003, \mathbf{8 5}(1)$ : 92-108.

[12] Feng Shuangshuang, Tian Bing, Hu Yincui et al. The hyper-spectra characteristics of degradation indicator species in the grassland of Bashang region. Journal of Arid Land Resources and Environment, 2016, 30(3): 133-139. [冯双双, 田冰, 胡引翠等. 坝上草原退化指示种光谱特征研究. 干旱区资源与环境,2016, 30(3)：133-139.]

[13] Chen Yonggang, Ding Lixia, Ge Hongli et al. Hyperspectral feature band selection based on mean confidence interval and tree species discrimination. Spectroscopy and Spectral Analysis, 2011, 31(9) : 2462-2466. [陈永刚, 丁丽霞, 葛宏立等. 基于均值置信区间带的高光谱特征波段与树种识别. 光谱学与光谱分析, 2011,31(9): 2462-2466.]

[14] Su Hongjun, Du Peijun. Study on feature selection and extraction of hyperspectral data. Remote Sensing Technology and Application, 2006,21(4): 288-293. [苏红军, 杜培军. 高光谱数据特征选择与特征提取研究. 遥感技术与应用, 2006,21(4) : 288-293.]

[15] Sheng Zhou, Xie Shiqian, Pan Chenyi eds. Probability and mathermatical statistics. Beijing: Higher Education Press, 2008: 161-169. [ 盛骤, 谢式千, 潘承毅. 概率论与数理统计. 北京: 高等教育出版社, 2008: 161-169.]

[16] Li Yurong, Xiang Guobo. A linear discriminant analysis classification algorithm based on mahalanobis distance. Computer Simulation, 2006, 23(8): 86-88. [李玉榕, 项国波. 一种基于马氏距离的线性判别分析分类算法. 计算机仿真, $2006,23(8): 86-88$.

[17] Wu Xianghua, Niu Shengjie, Wu Chenou et al. An improvement on estimating coveriance matrix during cluster analysis using mahalanobis distance. Journal of Applied Statistics and Management, 2011,30(2): 240-245. [吴香华, 牛生杰, 吴 诚鸥等. 马氏距离聚类分析中协方差矩阵度算的改进. 数理统计与管理, 2011, 30(2): 240-245.]

[18] Yu Xuchu, Feng Wufa, Yang Guopeng et al eds. Analysis and application of hyperspectral image. Beijing: Science Press, 2013: 12-14. [余旭初, 冯伍法, 杨国鹏等. 高光谱影像分析与应用. 北京: 科学出版社, 2013: 12-14.] 